\title{
Artérias da bolsa cloacal de galinhas (Gallus gallus) da linhagem Hybro PG
}

\author{
Andréa Regina Abrantes Gomes* \\ Frederico Ozanam Carneiro e Silva \\ Renata Lima Miranda \\ Gabrielle Gonçalves Narciso Resende
}

Faculdade de Medicina Veterinária, Universidade Federal de Uberlândia Rua Ceará s/n, Bloco D, Campus Umuarama, CEP 38405-382, Uberlândia - MG, Brasil

*Autor para correspondência

andreagomes85@gmail.com

Submetido em 19/03/2009

Aceito para publicação em 27/09/2009

\section{Resumo}

A pesquisa baseou-se no estudo da origem, número e ordenação dos vasos arteriais e seus ramos os quais irrigam a bolsa cloacal de 30 galinhas (Gallus gallus) da linhagem Hybro PG. Inicialmente, foi injetada, na artéria isquiática dos exemplares, uma solução aquosa a 50\% de Neoprene Látex 450 e, posteriormente, realizada a dissecação. Os resultados evidenciaram a participação das artérias pudendas interna esquerda e direita e bursocloacal, em todos os animais estudados, variando apenas o número e a ordenação de seus ramos. Houve também vasos oriundos das artérias caudal mediana (23\%) e pudenda externa esquerda (3,33\%). O número total de ramos variou de um a quatro e sua distribuição seguiu de forma singular para cada exemplar.

Unitermos: aves, bolsa cloacal, tecido linfóide, vascularização

\section{Abstract}

Arterial supply to the cloacal bursa of Hybro PG lineage hens (Gallus gallus). A study was carried out on the origin, number and disposition of the arterial vessels and their branches which irrigated the cloacal bursas of 30 hens (Gallus gallus) of the Hybro PG lineage. First of all, the left isquiadic artery from the samples was cannulated and injected with a $50 \%$ colored solution of Neoprene latex " 450 "; then, we continued the experiment with dissection whose results confirmed that the cloacal bursa was supplied by direct branches from the left and right internal pudendal arteries and bursocloacal arteries in $100 \%$ of the cases, varying only in terms of the numbers and disposition of their branches. There were also some branches from the median caudal artery $(23 \%)$ and left external pudendal (3.33\%). The total number of branches varied from 1 to 4 and their distribution was unique in each sample.

Key words: cloacal bursa, fowl, lymphoid tissue, vascularization 


\section{Introdução}

Pesquisadores e avicultores vêm investindo em estudos na área de manutenção da qualidade das raças e na prevenção de doenças infecto contagiosas, anomalias congênitas e distúrbios ligados ao sistema imunológico de aves de produção, de acordo com Silva et al. (1997).

Doenças como a doença infecciosa da bursa, relacionada à imunossupressão de aves domésticas, com alta taxa de mortalidade entre os jovens, vêm ao longo das últimas décadas ocasionando prejuízos econômicos à avicultura tanto brasileira quanto em âmbito mundial (Marinho et al., 2003). Tal doença acomete um dos principais órgãos do sistema imunológico desses animais, a bolsa cloacal, cuja função, juntamente com o timo, é a maturação e transferência de linfócitos para outros tecidos dependentes: baço, nódulos linfáticos agregados ao canal alimentar e glândula lacrimal da terceira pálpebra (Frazier, 1963).

O conhecimento sobre a bolsa cloacal e sua associação com o sistema imunológico é incompleto, sendo necessário um maior estudo de sua morfologia e fisiologia para poder elucidar problemas sobre o desempenho desta na imunidade das aves; portanto, muitos autores ao se referir à vascularização deste órgão acabam se tornando genéricos (Silva et al., 1997).

Santana et al. (2003) analisaram a vascularização arterial da bolsa cloacal em matrizes de corte Hubbard, onde concluíram ser o órgão irrigado por ramos diretos $\mathrm{e}$ indiretos, oriundos das artérias pudendas internas, direita e esquerda, além de ramos diretos das artérias caudal mediana, ilíaca interna esquerda e mesentérica caudal.

Silva et al. (1997) observaram, em matrizes da linhagem Ross, ramos diretos e indiretos das artérias pudendas internas direita e esquerda e caudal mediana.

Autores como Pintea et al. (1967) e Nickel et al. (1981) afirmam que as artérias pudendas internas direita e esquerda, cuja origem comum é a aorta abdominal, são as responsáveis pela irrigação daquele órgão, porém são inespecíficos com relação a seus ramos.

Pintea et al. (1967), no entanto, descrevem, em galinhas, o percurso dos vasos arteriais e venosos que chegam e saem da bolsa cloacal, onde ramos bursocloacais são originários das artérias pudendas internas comuns e mesentérica caudal. Ao passo que Nickel et al. (1981) descrevem as artérias pudendas internas originando-se das artérias pudendas comuns, bifurcando-se em ramos musculares e intestinais, dos quais os últimos vascularizam a cloaca e o órgão em estudo.

Durante estudo sobre a linhagem Label Rouge, Silva et al. (2003) verificaram que a bolsa cloacal recebe o seu suprimento vascular arterial, independentemente do número e do território de sua distribuição, a partir das artérias bursocloacais e cloacais, derivadas respectivamente das artérias pudendas internas, direita e esquerda; sendo possível a associação com ramos colaterais oriundos da artéria mesentérica caudal.

Autores como Santana et al. (1997) observaram, em aves da linhagem Peterson, a presença das artérias cloacais, além da participação das artérias bursocloacais, mesentérica caudal e caudal mediana.

Getty (1986) e Silva et al. (2003) evidenciam a irrigação da bolsa cloacal como sendo feita por ramos das artérias pudendas internas direita e esquerda, originadas das artérias ilíacas internas correspondentes.

Por sua vez, Zamojska (1975) este considera ainda a participação de ramos das artérias sacral mediana e pudendas internas na nutrição da bolsa cloacal.

Nas pesquisas de Santana et al. (2000), sobre a bolsa cloacal de aves (Gallus gallus) da linhagem Avian Farms, é descrita a vascularização desta por artérias bursocloacais, oriundas das artérias pudendas internas direita e esquerda, exclusivamente ou em associações com ramos bursais da artérias mesentérica caudal e caudal mediana.

O presente trabalho visa evidenciar a origem, o número e a distribuição do contingente arterial das aves (Gallus gallus) da linhagem Hybro PG que irrigam a bolsa cloacal, o qual apresenta uma significativa importância imunológica, pois doenças imunossupressoras que atingem a bolsa cloacal elevam a mortalidade, evidenciando a co-relação deste órgão com o sistema imunológico e portanto a necessidade de uma boa vascularização desse.

\section{Material e Métodos}

Utilizaram-se 30 galinhas da linhagem de corte Hybro PG, com idades variando entre 10 a 12 semanas, 
oriundas do matrizeiro da Universidade Federal de Uberlândia e Granja Rezende S/A, ambos localizados no município de Uberlândia - MG, após morte natural.

Com o objetivo de marcar os contingentes arteriais das aves, injetou-se, mediante canulação prévia da artéria isquiática direita, uma solução de Neoprene Látex "450” (Du Pont do Brasil S/A), diluída em água a $50 \%$ e corada com pigmento especifico (Globo S.A Tintas e Pigmentos), em seguida as peças foram fixadas em solução aquosa de formol a $10 \%$, pela aplicação tanto intracavitária como pela imersão em recipientes contendo a mesma solução, durante um período de no mínimo 48 horas, para posterior dissecação.

Para a dissecação foram realizadas duas incisões paramedianas na base da cauda e, divulsionando-se entre esta e a abertura cloacal, foi possível alcançar as artérias responsáveis pela irrigação da bolsa cloacal, após a retirada dos tecidos adiposo e conjuntivo que recobriam a mesma.

Os modelos de vascularização arterial das bolsas cloacais foram transferidos mediante esquemas representativos, para fichas individuais, registrando a origem, o número e a ordenação dos componentes arteriais.

Após as dissecações foram realizadas fotografias para ulterior documentação (Figuras 1 e 2).

\section{Resultados}

As artérias pudendas internas esquerda e direita são responsáveis pelo suprimento dos 30 casos analisados $(100 \%)$ em associação ou não com as artérias caudal mediana, a qual contribui com ramos para $20 \%$ dos exemplares, e bursocloacais esquerda e direita, com $60 \%$ e $43,33 \%$, respectivamente; as artérias ilíacas internas, tanto esquerda como direita, contribuíram para a irrigação da bolsa cloacal em 3,33\% das aves dissecadas (Tabela 1).

Os ramos arteriais se dividem antes de penetrar no parênquima do órgão pelas faces ventral, dorsal, laterais e caudal.

Quanto ao número de ramos pode-se evidenciar uma variação de um a três. A artéria pudenda interna esquerda emitiu um ramo em 19 casos (63,33\%), dois em 10 casos (33,34\%) e três em um exemplar (3,33\%); em contrapartida, sua correspondente direita enviou um ramo em 20 casos $(66,66 \%)$ e dois em 10 casos (33,34\%) (Tabela 1$)$.

A artéria caudal mediana contribuiu com um ou dois ramos nos seis exemplares, sendo um ramo em cinco casos e dois ramos em um espécime (Tabela 1).

TABELA 1: Frequência absoluta de casos que apresentam (x) número de ramos destinados à bolsa cloacal de galinhas da linhagem Hybro PG, de acordo com sua origem.

\begin{tabular}{cccc} 
& \multicolumn{3}{c}{ Número de ramos } \\
\cline { 2 - 4 } Artérias & $\mathbf{1}$ & $\mathbf{2}$ & $\mathbf{3}$ \\
\hline ABCE & 11 & 7 & - \\
ABCD & 11 & 2 & - \\
APIE & 19 & 10 & 1 \\
APID & 20 & 10 & - \\
ACM & 5 & 1 & - \\
AIIE & 1 & - & - \\
AIID & 1 & - & - \\
\hline
\end{tabular}

$\mathrm{ABCE}$ - artéria bursocloacal esquerda; $\mathrm{ACM}$ - artéria caudal mediana; ABCD - artéria bursocloacal direita; AIIE - artéria ilíaca interna esquerda; APIE - artéria pudenda interna esquerda; AIID - artéria ilíaca interna direita; APID - artéria pudenda interna direita.

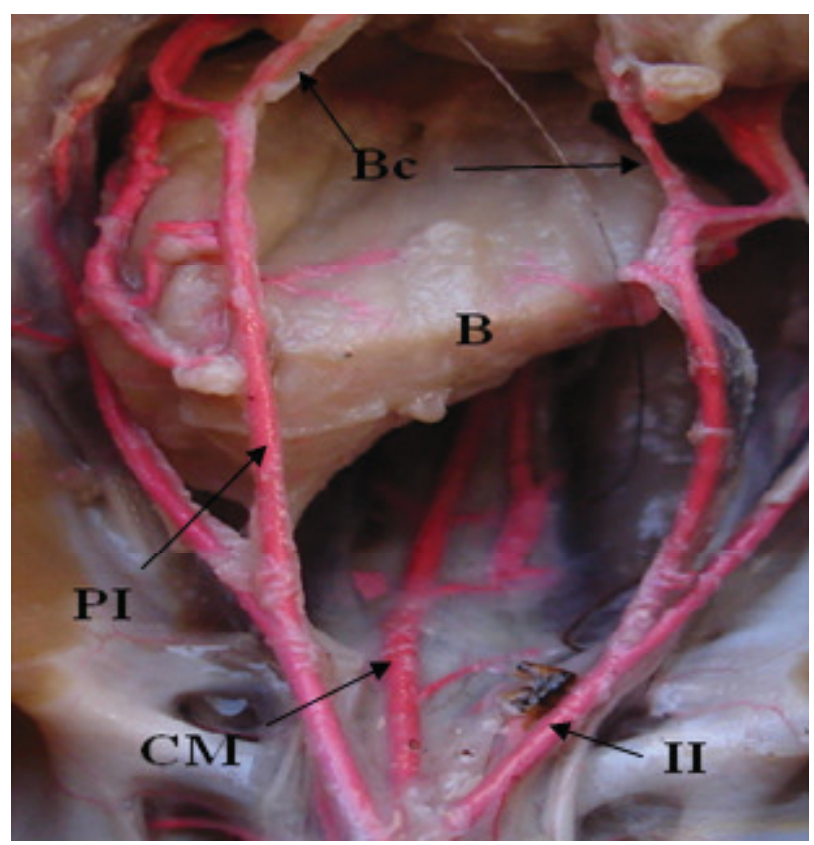

FIGURA 1: Nesta fotografia observam-se os ramos arteriais que indiretamente ou diretamente irrigam a bolsa cloacal (B). Artéria ilíaca interna (II), artéria pudenda interna (PI), artéria caudal mediana (CM) e artéria bursocloacal (Bc). 


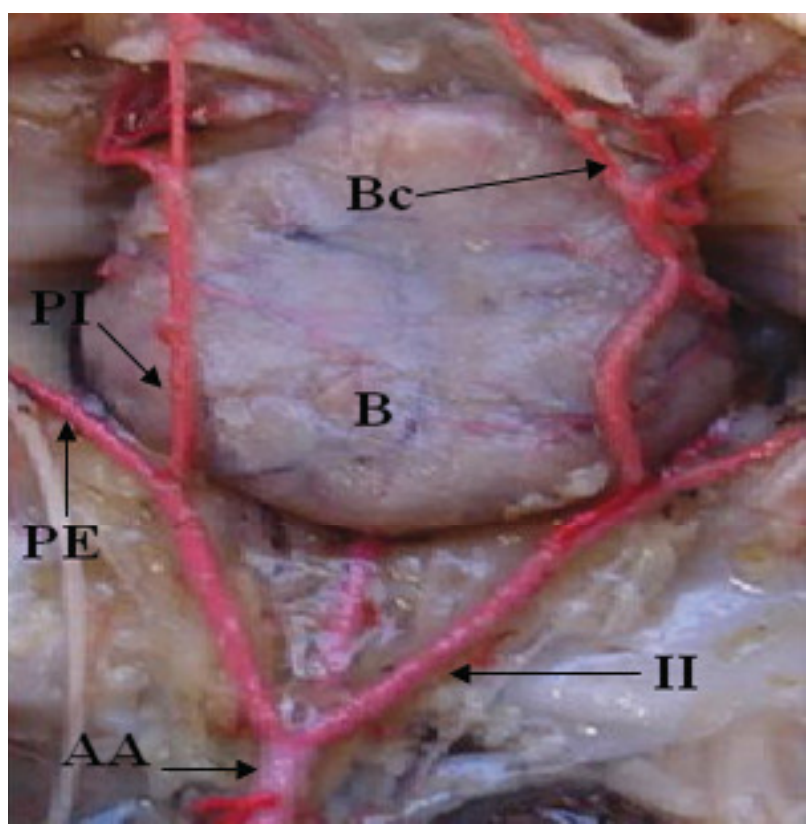

FIGURA 2: Representação fotográfica da bolsa cloacal (B) sendo irrigada por ramos das artérias pudenda interna (PI), bursocloacais $(\mathrm{Bc})$, aorta abdominal (AA), pudenda externa (PE) e ilíaca interna (II).

\section{Discussão}

Trabalhos realizados por Santana et al. (1997; 2000; 2003) e Silva et al. (1997; 2003) relatam a origem, o número e a ordenação das artérias destinadas à nutrição da bolsa cloacal, órgão esse de significativa importância imunológica, em aves das linhagens Peterson, Avian Farms, Hubbard, Ross e Label Rouge, respectivamente.

No que diz respeito à origem das artérias, Nickel et al. (1981) e Pintea et al. (1967) afirmam que as artérias pudendas internas comuns, termo empregado como sinonímia para artéria ilíaca interna, originadas da aorta abdominal, ao se bifurcarem vão formar as artérias pudendas internas as quais são responsáveis pela irrigação do órgão em questão, porém não se detêm na especificação de seus ramos; ao passo que Getty (1986), Santana et al. (1997; 2000; 2003) e Silva et al. (1997; 2003) afirmam que a origem das artérias pudendas internas se dá a partir das artérias ilíacas internas, fato também evidenciado neste trabalho.

Pintea et al. (1967), Santana et al. (1997; 2000; 2003) e Silva et al. $(1997 ; 2003)$ relatam a participação das artérias bursocloacais oriundas das artérias pudendas internas direita e esquerda na vascularização da bolsa cloacal, o qual esta de acordo com o presente trabalho.

No entanto, não foi comprovada, neste trabalho, a participação da artéria mesentérica caudal, em associação ou não com a artéria caudal mediana, na irrigação do órgão, conforme descrito por Santana et al. (1997; 2000; 2003), Silva et al. (1997; 2003) e Zamojska (1975), ressalte-se que este último autor utiliza o termo artéria sacral mediana referindo-se à artéria caudal mediana. Santana et al. (2003) fazem referência ao termo artéria ilíaca esquerda como sinonímia de artéria ilíaca interna esquerda.

As artérias cloacais, originadas das artérias pudendas internas, também representam parte da nutrição da bolsa cloacal, conforme descrevem Santana et al $(1997 ; 2000)$ Silva et al. $(1997 ; 2003)$.

Em suma, quando considerado a origem, o número e a disposição total de vasos em cada espécime estudado, o comportamento vascular apresenta disposição própria para cada observação, de acordo com Santana et al. (1997; 2000; 2003) e Silva et al. (1997; 2003), o que também foi constatado neste experimento.

Assim, pode-se concluir que a bolsa cloacal de matrizes de corte da linhagem Hybro PG é irrigada por ramos arteriais pelos ramos oriundos das artérias pudendas internas, esquerda e direita, bursocloacais direita e esquerda, podendo estar ou não em associação com ramos das artérias caudal mediana e ilíacas internas esquerda e direita. Além disso, ao considerar a origem e a quantidade de vasos que suprem a bolsa cloacal, o comportamento vascular se mostrou particular em cada caso analisado.

\section{Referências}

Frazier, J. 1963. The ultraestructure of lymphoid follicles of the chick bursa of Fabricius. Acta Anatomica, 113: 1-7.

Getty, R. 1986. Sisson/Grossman anatomia dos animais

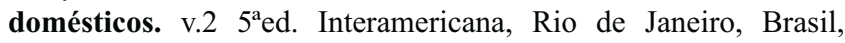
2000pp.

Marinho, C. E.; Fernandes, M. J. B.; Simoni, I. C. 2003. Estudo do ciclo de replicação do vírus da doença infecciosa das bursas de galinha na linhagem celular RK-13. Arquivo do Instituto Biológico, 70 (1): 5-9. 
Nickel, R.; Schummer, A.; Seiferle, E. 1981. Anatomy of the domestic birds. v.4. Verlag Paul Parey, Berlin, Germany, 148pp.

Pintea, V.; Constantinescu, G. U.; Radu, C. 1967. Vascular and nervous supply of the bursa of Fabricius in the hen. Acta Veterinary Science, 17: 263-268.

Santana, M. I. S.; Silva, F. O. C.; Severino, R. S.; Santos, A. L. Q.; Bombonato, O. P. P.; Marçal, A. V. 2003. Vascularização da bolsa cloacal em Gallus gallus domesticus (matrizes de corte Hubbard). Brazilian Journal of Veterinary Research and Animal Science, 40 (4): 254-260.

Santana, M. I. S.; Silva, F. O. C.; Severino, R. S.; Santos, A. L. Q.; Drummond, S. S.; Bombonato, O. P. P. 1997. Vascularização arterial da bolsa cloacal em aves da linhagem Peterson (Gallus gallus domesticus). Anais do $\mathbf{2 5}^{\circ}$ Congresso Brasileiro de Medicina veterinária, Gramado, Brasil, p.106.

Santana, M. I. S.; Silva, F. O. C.; Severino, R. S.; Santos, A. L. Q.; Drummond, S. S.; Bombonato, O. P. P. 2000. Vascularização arterial da bolsa cloacal em Gallus gallus domesticus (Matrizes de corte Avian Farms). Brazilian Journal of Veterinary Research and Animal Science, 35 (2): 115-120.
Silva, F. O. C.; Severino, R. S.; Drummond, S. S.; Machado, G. V.; Bombonato, P. P.; Santana, M. I. S.; Lima, E. M. M. 2003. Suprimento arterial para a bolsa cloacal de galinhas (Gallus gallus Linnaeus, 1758) da linhagem Label Rouge. Archives of Veterinary Science, 8 (1): 13-18.

Silva, F. O. C.; Severino, R. S.; Santos, A. L. Q.; Drummond, S. S; Bombonato, P. P.; Santana, M. I. S; Gonçalez, P. O; Marçal, A. V. 1997. Vascularização arterial da bolsa cloacal em Gallus gallus domesticus (linhagem Ross). Revista da Faculdade de Zootecnia, Veterinária e Agronomia, 4 (1): 81-92.

Zamosjka, D. 1975. Anatomical studies on the vascularization of the bursa of Fabricius and Uropigeal gland in the hens (Gallus domesticus). Part II. Blood vessels of the bursa of Fabricius (Bursa of Fabricii). Zoologica Poloniae, 24 (3/4): 455-476. 